\title{
2D or not 2D?
}

\author{
Flat is the question.
}

\begin{abstract}
Whether 'tis nobler in the mind to suffer
The slings and arrows of the outrageous limitations of graphene,

Or to take arms against a sea of ordered anthracenes,

And by photoirradiation polymerize them?
\end{abstract}

Polymers come in many different forms and are used in a huge variety of applications, from the mundane to the highly specialized. Naturally occurring biopolymers such as DNA and proteins underpin life itself, whereas synthetic polymers such as polyolefins and polyesters have played a crucial role in shaping the modern world. And what they all have in common is that they consist of repeating units - sometimes just one type and sometimes a collection of related building blocks. Conceptually, the simplest polymer architecture is one in which each repeat unit is linked to two neighbours to form a linear chain, and can be thought of as a 'one-dimensional' structure. But what of higher dimensions?

Two-dimensional polymers are sheetlike, covalently bonded molecular tilings that extend in exactly two dimensions ${ }^{1,2}$. Unlike 1D polymers, over which chemists can often exert high levels of control during their synthesis, the design and production of $2 \mathrm{D}$ polymers presents far greater challenges. Graphene is perhaps the archetypal 2D material, and although it has enjoyed a great deal of attention, the idea of precisely tailoring its size, shape and functionality at the molecular level seems somehow incompatible with something that can be made in a kitchen blender ${ }^{3}$. Perusing the recent literature, you could be forgiven for thinking that graphene was the only $2 \mathrm{D}$ material in existence, but other natural and synthetic layered substances have also been exfoliated into $2 \mathrm{D}$ sheets ${ }^{4}$.

The unique optical and electronic properties that arise from the $2 \mathrm{D}$ structure of graphene have attracted the attention of many researchers, including those interested in modulating its behaviour through chemical functionalization - although there can be some uncertainty regarding the exact nature and placement of functional groups. Bottomup approaches to functional graphene fragments ${ }^{5}$ (or so-called nanographenes) look to overcome the problems associated with top-down methods, but are currently limited in terms of the size of sheets that can be formed, and the functionality that can be introduced. Two-dimensional synthetic polymers arguably have the potential to overcome these limitations, particularly if large crystals can be grown. Single crystals of 3D framework materials - including fully organic ones ${ }^{6}$ - have been reported, indicating a free-standing 2D analogue to be a realistic target.

Now, two Articles in this issue (page 774 and page 779) describe the formation of $2 \mathrm{D}$ polymers - through the photodimerization of rigid anthracene-based monomers - in a single-crystal-to-single-crystal fashion. Although 2D polymers have been synthesized before by topochemical solidstate polymerization ${ }^{7}$, and $2 \mathrm{D}$ covalentorganic frameworks have also been made on surfaces and in thin films ${ }^{8}$, these reports represent the first detailed characterization of the $2 \mathrm{D}$ polymerization process, where the monomer, intermediate states and polymer all retain their crystallinity. In what has been designated as the International Year of Crystallography, here are two more examples of the importance and power of crystallography in chemistry research ${ }^{9}$.

As all scientists are aware - sometimes painfully so - there is a lot more behind a paper than just the words and figures in the published article. In an Interview in this issue (page 751) the leaders of the two groups that carried out the work, Benjamin King and Dieter Schlüter, offer some interesting insights into their motivation and background to the story. In an age where 'arms races' between competing groups so often exist, King and Schlüter decided to go against the norm; King saying: "while we do compete, our collaboration is synergistic, and our openness to helping one another has advanced the field more than if we had struggled alone." Not only did they coordinate their submissions to Nature Chemistry, but they also agreed that King's paper should appear first in the print issue because his group obtained their 2D-polymer single-crystal data first.

Whereas coordinating the publication of their papers turned out to be relatively straightforward, the work that got them to that point certainly wasn't. Schlüter explains: "Patrick Kissel's first synthesis of a 2DP during his PhD was horribly complicated. A 25-step synthetic procedure sounds more like natural product than polymer synthesis and surely discourages anyone from following it." Nevertheless, they persisted, and "the monomer used in our current Nature Chemistry paper is now available in three steps (even though five steps are described in the manuscript, which was correct at the time of submission) from cheap commercially available chemicals."

In an accompanying News and Views article (page 757), Neil Champness explains why we should be excited about this class of material: "consider a molecular-scale membrane comprising highly organized components with specific properties be they optical, magnetic or electronic and the ability to recognize specific target molecules through the incorporation of tailored recognition sites. Sounds like a chemist's dream!" Given the progress that has been made towards making single-crystal sheets of $2 \mathrm{D}$ polymers from readily available starting materials, he seems justified in saying that "the future of these materials is undoubtedly bright and I have no doubt that this is a field that will grow and grow."

The obvious next steps are to make larger sheets, and to introduce functionalities on one or both sides. Increasing the range of monomers that can be stitched together to form $2 \mathrm{D}$ sheets through different polymerization chemistries would significantly broaden the range of materials that could be targeted. For 2D polymers to be considered viable for practical purposes, however, their production needs to be scalable so that they can be made in large enough quantities - at present, designing and making the monomer is arguably the limiting step. Future developments will hopefully pave the way for $2 \mathrm{D}$ polymers to be as diverse and widely used as their $1 \mathrm{D}$ counterparts, and one day they may even be as popular - and headline-generating — as graphene.

\footnotetext{
References

1. Sakamoto, J., van Heijst, J., Lukin, O. \& Schlüter, A. D. Angew. Chem. Int. Ed. 48, 1030-1069 (2009).

2. Colson. J. W. \& Dichtel, W. R. Nature Chem. 5, 453-465 (2013)

3. Paton, K. R. et al. Nature Mater. 13, 624-630 (2014).

4. Novoselov, K. S. et al. Proc. Natl Acad. Sci. USA 102, 10451-10543 (2005).

5. Chen, L., Hernandez, Y., Feng, X. \& Müllen, K. Angew. Chem. Int Ed. 51, 7640-7654(2012).

6. Beaudoin, D., Maris, T. \& Wuest, J. D. Nature Chem. 5, 830-834 (2013).

7. Kissel, P. et al. Nature Chem. 4, 287-291 (2012).

8. Colson, J. W. et al. Science 332, 228-231 (2011).

9. http://www.nature.com/milestones/milecrystal/index.html
} 